\title{
Efeito do ensacamento de cachos de banana 'Nanicão' na produção e no intervalo entre inflorescência e colheita ${ }^{(1)}$
}

\author{
José Nilton Medeiros Costa(2), João Alexio Scarpare Filho ${ }^{(3)}$ e Ricardo Alfredo Kluge ${ }^{(4)}$
}

\begin{abstract}
Resumo - O objetivo deste trabalho foi avaliar o efeito do ensacamento de cachos de bananas (Musa sp. AAA), cultivar Nanicão, em diferentes épocas, na produção e no intervalo entre inflorescência e colheita. $\mathrm{O}$ experimento foi realizado no Município de Tietê, SP, em blocos ao acaso, em esquema fatorial $2 \times 3$, com quatro repetições. O ensacamento foi efetuado com sacos de polietileno em três épocas: 21/5/96, 17/12/96 e 26/2/97. Foram avaliados o intervalo entre emergência da inflorescência e colheita, a massa do cacho e o comprimento, diâmetro e densidade do fruto. A interação ensacamento x épocas não foi significativa em relação a nenhuma das variáveis. O ensacamento diminuiu o intervalo entre a emergência da inflorescência e a colheita na primeira (21/5/96) e segunda época (17/12/96).
\end{abstract}

Termos para indexação: Musa, cobertura de plástico, fruta, qualidade, manejo de cultura.

\section{Effect of bagging bunches of 'Nanicão' banana in the production and in the interval between inflorescence and harvest}

\begin{abstract}
The objective of this work was to evaluate the effect of bagging bunches of 'Nanicão' banana (Musa sp. AAA), at different times, in the production and in the interval between the inflorescence and harvest. The experiment was carried out in Tietê county, SP, Brazil, in randomized blocks, and arranged in a $2 \times 3$ factorial with four repetitions. Bagging was done with polyethylene sacks at three times (May 21 and December 17, 1996 and February 26, 1997). The interval between inflorescence and harvest, mass of the bunch and the length, diameter and density of the fruit were appraised. No significance was found in the interaction bagging $\mathrm{x}$ times. Bagging decreased the interval between the emergency of the inflorescence and harvest in the first and second time.
\end{abstract}

Index terms: Musa, plastic film cover, fruit, quality, crop management.

\section{Introdução}

$\mathrm{O}$ acondicionamento em sacos de plástico de cachos de banana, em início de desenvolvimento, pode contribuir para aumento da massa dos cachos, redução do intervalo entre a emergência da inflorescência e a colheita, melhoria visual dos frutos, aumento do

\footnotetext{
(1) Aceito para publicação em 9 de julho de 2002. Extraído da dissertação de mestrado apresentada pelo primeiro autor à Escola Superior de Agricultura Luiz de Queiroz (Esalq), Piracicaba, SP.

(2) Embrapa-Centro de Pesquisa Agroflorestal de Rondônia, BR 364, km 5,5, Caixa Postal 406, CEP 78900-970 Porto Velho, RO. E-mail: jnilton@cpafro.embrapa.br

${ }^{(3)}$ Esalq, Dep. de Horticultura, Caixa Postal 9, CEP 13418-900 Piracicaba, SP. E-mail: jascarpa@ carpa.ciagri.usp

(4) Esalq, Dep. de Ciências Biológicas. E-mail: rakluge@carpa.ciagri.usp.br
}

tamanho final do fruto, proteção contra o frio, ação abrasiva das folhas e ataque de insetos (Heenan, 1973; Soto Ballestero et al., 1992; Lichtemberg, 1996).

Os resultados de diferentes pesquisas sobre o efeito da redução do intervalo entre a emergência da inflorescência e a colheita no aumento da produção nem sempre estão em concordância.

Em relação à massa do cacho, foram registrados, em bananas 'Dwarf Cavendish', aumentos de 25\% por Cann (1965) na Austrália, e 16,7\% na África do Sul por Robinson \& Nel (1984). Em três localidades do Vale do Aridane (Ilhas Canárias), caracterizadas por apresentarem altitudes diversas, Galán Saúco et al. (1996) verificaram em 'Dwarf Cavendish' aumentos de $8,7 \%, 14,2 \%$ e $18,2 \%$ na massa do cacho, dos quais os dois valores maiores foram significativos e correspondem às localidades de média e alta altitude, respectivamente. Por outro lado, Sampaio \& 
Simão (1970), Daniells et al. (1992) e Salomão (1995), nas cultivares Nanica, Williams e Mysore, respectivamente, não encontraram diferenças em relação a massa de cachos ensacados e não ensacados.

Quanto ao intervalo entre a inflorescência e a colheita, Berril (1956), Turner \& Rippon (1973) e Daniells et al. (1987) constataram um intervalo mais curto nos cachos ensacados, quando comparados com os cachos não ensacados. Robinson \& Nel (1984) não encontraram diferenças, enquanto Scott et al. (1971) observaram um maior intervalo em relação aos cachos ensacados.

As contradições apresentadas em relação ao intervalo entre a emergência da inflorescência e a colheita estão relacionadas aos critérios usados na definição do ponto de colheita, cuja determinação pode ser subjetiva. Podem ser também, conseqüência da utilização de sacos abertos ou fechados na extremidade distal dos cachos (Galán Saúco et al., 1996).

O ensacamento de cachos em sacos de polietileno é prática efetuada em muitas outras regiões produtoras de banana do mundo (Soto Ballestero, 1992; Galán Saúco et al., 1996), sendo rotineira em bananais cultivados por grandes empresas agrícolas em países da América Central (Moreira, 1987), desde a década de 60 (Perumal \& Adam, 1968). No Brasil, esta prática tem sido executada em caráter experimental e, recentemente, tem sido adotada por alguns produtores nos estados de São Paulo e Santa Catarina, embora as informações sobre a técnica sejam ainda limitadas com relação às diferenças entre cultivares e quanto às condições climáticas.

O objetivo deste trabalho foi avaliar o efeito do ensacamento de cachos de bananas (cultivar Nanicão) em diferentes épocas, na produção, e no intervalo entre a emergência da inflorescência e a colheita.

\section{Material e Métodos}

O experimento foi realizado na Fazenda Diamante, Município de Tietê, SP, com coordenadas de $23^{\circ} 7^{\prime}$ latitude Sul e $47^{\circ} 43^{\prime}$ longitude Oeste. O clima da região é classificado como Cwa: tropical de altitude, com inverno seco e verão chuvoso. Os dados de temperatura e precipitação pluvial dos anos de 1996 e 1997 são apresentados na Figura 1 . A cultivar utilizada foi a Nanicão. O bananal foi esta- belecido no espaçamento de 2,10 x 2,40 m e estava, na ocasião da instalação do experimento, com três anos de idade. $\mathrm{O}$ delineamento experimental usado foi o de blocos ao acaso, em esquema fatorial $2 \times 3$ com quatro repetições, sendo cada parcela composta por três plantas. Os tratamentos consistiram de cachos ensacados, cachos não ensacados e de três épocas de ensacamento (21/5/96, 17/12/96 e 26/2/97).

Antes da instalação do ensaio, estudou-se a homogeneidade das plantas, a fim de selecioná-las quanto ao número de pencas e tamanho do cacho. Determinou-se como padrão a seleção de bananeiras com cachos de sete pencas. Esse número foi estabelecido em razão da ocorrência do maior porcentual de plantas que apresentavam esta característica.

Conforme a casualização das parcelas, os cachos selecionados foram ensacados com sacos de plástico de coloração azul, com $1,45 \mathrm{~m}$ de altura por $0,74 \mathrm{~m}$ de largura e $0,08 \mathrm{~mm}$ de espessura, com furos de $10,0 \mathrm{~mm}$ de diâmetro, distribuídos em quadrados a cada $85 \mathrm{~mm}$.

Antes do ensacamento procedeu-se à retirada do coração e a despistilagem nos frutos, respectivamente, de ambos os tratamentos (cachos ensacados e cachos não ensacados). A extremidade distal da raquis floral masculina foi quebrada a $10-12 \mathrm{~cm}$ do ponto de inserção da última penca feminina e descartada, segundo Moreira (1987). Os sacos foram amarrados com fita plástica no engaço, na parte imediatamente superior da primeira cicatriz bracteal, conforme Soto Ballestero et al. (1992).

As variáveis estudadas foram: a) intervalo entre a emergência da inflorescência e a colheita (I-C); estabeleceu-se como limite para este intervalo o calibre de $34 \mathrm{~mm}$ nos frutos da segunda penca inferior, por ser equivalente, nas condições do experimento, ao ponto de colheita para o mercado interno, quando as bananas podem ser colhidas em estádio mais avançado (Bleinroth, 1985; Moreira, 1987); b) massa do cacho $(\mathrm{kg})$ obtida pela pesagem do cacho em balança com capacidade de $150 \mathrm{~kg}$; c) comprimento médio dos frutos $(\mathrm{cm})$ da segunda penca superior, considerada como representativa do cacho, para efeito de uniformização de amostras (Carvalho et al., 1989; Galán Saúco et al., 1996); as medições foram feitas com trena metálica flexível, na face côncava das bananas; d) diâmetro médio dos frutos $(\mathrm{mm})$ da segunda penca superior medido com paquímetro na região central dos frutos, perpendicularmente ao maior eixo; e) densidade dos frutos $\left(\mathrm{g} / \mathrm{cm}^{3}\right)$ obtida pela pesagem de três buquês com seis frutos verdes por repetição, os quais foram imersos em recipiente contendo água, para determinação do volume dos frutos, mediante o deslocamento da água; a densidade foi estabelecida pelo quociente massa/volume. 
$\mathrm{Na}$ avaliação das variáveis, os frutos foram amostrados da segunda à quarta pencas proximais de cada cacho (Salomão, 1995). Os cachos foram colhidos quando os frutos da fileira distal da segunda penca inferior atingiram o diâmetro de $34 \pm 1 \mathrm{~mm}$, medidos em suas regiões equatoriais. O ponto de colheita guardou relação com a prática adotada para o comércio interno, em razão dos frutos apresentarem equivalência nas condições do experimento. Os cachos foram colhidos, pesados e despencados pela manhã. As pencas selecionadas foram transportadas de imediato para as avaliações referentes ao comprimento, diâmetro e densidade dos frutos.

Os dados obtidos foram submetidos à análise de variância, mediante o Sistema SANEST, aplicando-se o teste $\mathrm{F}$, e a comparação das médias feitas pelo teste de Tukey a $5 \%$ de probabilidade.

\section{Resultados e Discussão}

O intervalo entre a inflorescência e a colheita (I-C) não apresentou diferença significativa quanto à interação ensacamento x época, no entanto, diferiu em relação aos fatores ensacamento e época (Tabela 1).

Em relação à época de ensacamento, como era esperado para I-C, o intervalo médio foi maior em 21/5/96 (176 dias), menor em 17/12/96 (115 dias) e intermediário em 26/2/97 (153 dias) (Tabela 1). Houve diferença entre cachos ensacados e não ensacados na primeira (21/5/96) e segunda épocas (17/12/96), ocorrendo uma redução do intervalo (I-C) para ca- chos ensacados de 9 e 11 dias, respectivamente. Berril (1956), Perumal \& Adam (1968), Turner \& Rippon (1973), Daniells et al. (1987) e Salomão (1995) também encontraram menores períodos entre a emergência da inflorescência e a colheita, em cachos ensacados.

A inferioridade do I-C em relação aos cachos ensacados em 21/5/96 pode ser por causa da elevação de temperatura durante o estádio final de crescimento e maturação fisiológica dos frutos, que ocorreu entre os meses de setembro e outubro/96 (Figura 1). Perumal \& Adam (1968) verificaram efeito do ensacamento sobre I-C mais pronunciado no inverno e salientaram que a colheita foi efetuada nessa estação, em condições de frio e chuvas. Segundo esses autores, tal comportamento é consequiência da elevação da temperatura no interior dos sacos, ocorrendo próximo às pencas superiores uma diferença de $1,7^{\circ} \mathrm{C}$ entre frutos ensacados e não ensacados. $\mathrm{O}$ ar mais quente concentrado na parte superior dos sacos parece ter sido o principal fator acelerador do crescimento das pencas superiores.

Todo o ciclo de desenvolvimento do cacho ensacado em 17/12/96 aconteceu nos meses mais quentes do ano, portanto, como já era esperado, o I-C foi menor nos cachos ensacados. Johns \& Scott (1989) comprovaram, mediante comparação de vários tipos de sacos usados na proteção de cachos, que ocorreu aumento de temperatura dentro dos
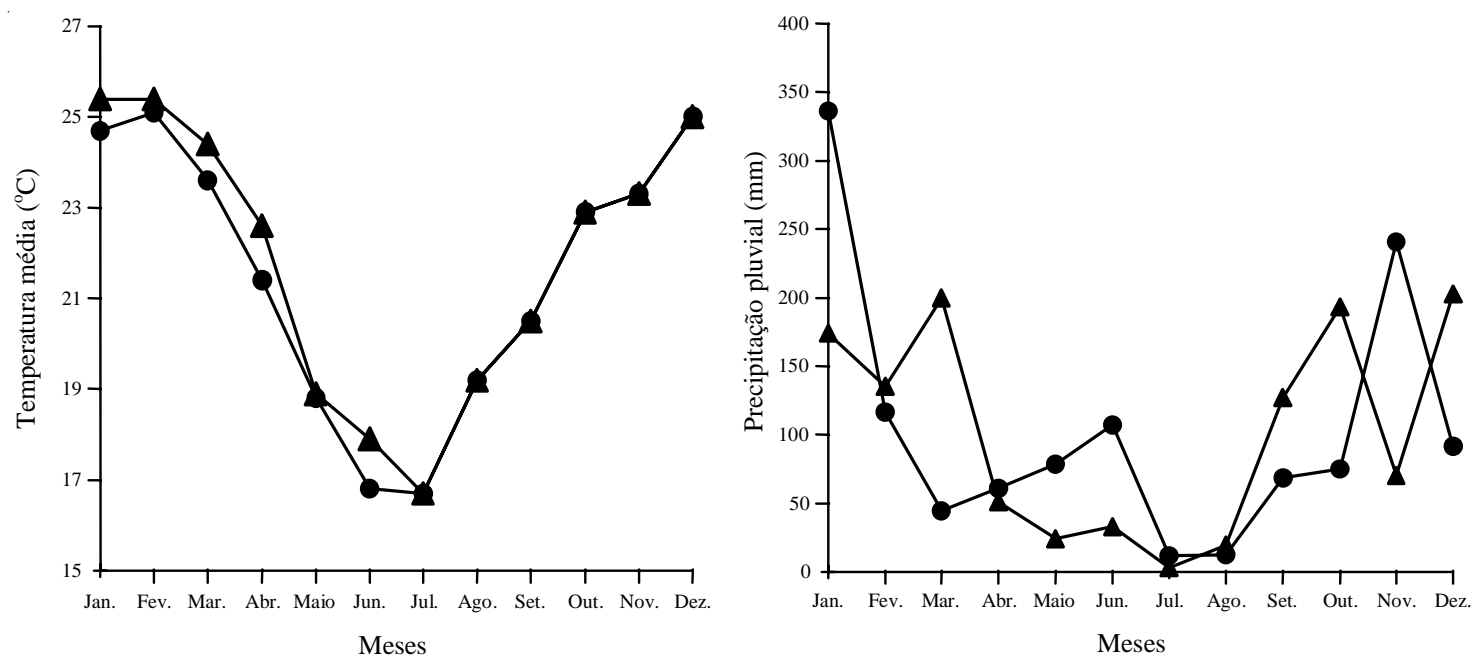

Figura 1. Temperatura média mensal e precipitação pluvial nos anos de 1996 (ム) e 1997 (•), em Tietê, SP. 
mesmos, e que tais temperaturas foram mais elevadas no verão. Há um consenso sobre o fato de que o aumento da temperatura, em torno de $1^{\circ} \mathrm{C}$, verificado em razão do ensacamento, reduz o intervalo I-C (Perumal \& Adam, 1968; Ganry, 1975; Robinson \& Nel, 1984; Johns \& Scott, 1989; Salomão, 1995).

O intervalo I-C observado em 26/2/97 não diferiu entre cachos ensacados e não ensacados. As bananeiras submetidas aos tratamentos em 26/2/97 tiveram as melhores condições climáticas para o desenvolvimento e produção, podendo-se inferir que o efeito do ensacamento depende tanto da época de realização quanto das condições climáticas do período. Resultados semelhantes aos obtidos em 26/2/97 foram constatados por Robinson \& Nel (1984) e Galán Saúco et al. (1996).

Em relação à massa dos cachos, houve significância apenas em relação ao fator época, com a maior média de massa observada em 26/2/97, diferente significativamente das médias obtidas em 21/5/96 e 17/12/96, as quais por sua vez não diferiram entre si (Tabela 1).
As diferenças de massa do cacho verificadas nas diferentes épocas podem ser explicadas pelas condições climáticas que ocorreram durante o desenvolvimento das plantas, formação e frutificação dos cachos (Figura 1). A temperatura e a precipitação são fatores de relevante importância no cultivo da bananeira, pois produções elevadas são obtidas em condições de temperatura elevada e uniforme, bem como permanente disponibilidade de umidade no solo (Alves et al., 1997; Kluge, 1998). A temperatura ótima para o desenvolvimento da bananeira situa-se em torno de $26^{\circ} \mathrm{C}$ (Medina, 1985).

A produção da primeira época (21/5/96) sofreu conseqüências de estiagem e baixas temperaturas. A segunda época (17/12/96) foi precedida por período de poucas chuvas e baixas temperaturas (abril a agosto/96), que coincidiu com a fase de desenvolvimento vegetativo e possivelmente com o início da diferenciação floral. Segundo Medina (1985), a quantidade de chuva exigida pela bananeira varia de 3 a $8 \mathrm{~mm}$ por dia. Portanto, no referido período, a precipitação mensal foi inferior ao limite mínimo necessá-

Tabela 1. Intervalo médio entre a emergência da inflorescência e a colheita, massa do cacho, comprimento e diâmetro dos frutos da segunda penca superior e densidade dos frutos em razão dos fatores ensacamento e épocas do ano em banana 'Nanicão'. Tietê, SP, $1997^{(1)}$.

\begin{tabular}{|c|c|c|c|c|}
\hline Tratamento & $21 / 5 / 96$ & $17 / 12 / 96$ & $26 / 2 / 97$ & Média \\
\hline & \multicolumn{4}{|c|}{ Intervalo entre a emergência da inflorescência e colheita (dias) } \\
\hline Ensacado & 171 & 109 & 153 & $144 \mathrm{~b}$ \\
\hline Não ensacado & 180 & 120 & 153 & $151 \mathrm{a}$ \\
\hline \multirow[t]{2}{*}{ Média } & $176 \mathrm{~A}$ & $115 \mathrm{C}$ & $153 \mathrm{~B}$ & \\
\hline & \multicolumn{4}{|c|}{ Massa do cacho $(\mathrm{kg})$} \\
\hline Ensacado & 14,45 & 16,20 & 20,91 & $17,19 \mathrm{a}$ \\
\hline Não ensacado & 14,42 & 15,03 & 19,42 & $16,29 \mathrm{a}$ \\
\hline \multirow[t]{2}{*}{ Média } & $14,44 \mathrm{~B}$ & $15,61 \mathrm{~B}$ & $20,17 \mathrm{~A}$ & \\
\hline & \multicolumn{4}{|c|}{ Comprimento do fruto $(\mathrm{cm})$} \\
\hline Ensacado & 18,99 & 22,38 & 21,69 & $21,02 \mathrm{a}$ \\
\hline Não ensacado & 18,96 & 21,94 & 20,94 & $20,61 \mathrm{a}$ \\
\hline \multirow[t]{2}{*}{ Média } & $18,97 \mathrm{~B}$ & $22,16 \mathrm{~A}$ & $21,31 \mathrm{~A}$ & \\
\hline & \multicolumn{4}{|c|}{ Diâmetro do fruto $(\mathrm{mm})$} \\
\hline Ensacado & 35,00 & 37,31 & 36,61 & $36,42 \mathrm{a}$ \\
\hline Não ensacado & 34,25 & 35,91 & 36,53 & $35,43 \mathrm{a}$ \\
\hline \multirow[t]{2}{*}{ Média } & $34,63 \mathrm{~A}$ & $36,61 \mathrm{~A}$ & $36,53 \mathrm{~A}$ & \\
\hline & \multicolumn{4}{|c|}{ Densidade do fruto $\left(\mathrm{g} / \mathrm{cm}^{3}\right)$} \\
\hline Ensacado & 1,002 & 0,999 & 0,998 & $0,999 \mathrm{a}$ \\
\hline Não ensacado & 1,006 & 1,001 & 0,995 & $1,001 \mathrm{a}$ \\
\hline Média & $1,004 \mathrm{~A}$ & $1,000 \mathrm{~A}$ & $0,996 \mathrm{~A}$ & \\
\hline
\end{tabular}


rio requerido pela cultura (Figura 1). A mudança da fase de desenvolvimento vegetativo do meristema apical para o estádio floral geralmente ocorre de três a quatro meses antes da emergência da inflorescência no ápice da planta (Turner, 1972a, 1972b), podendo chegar a mais de seis meses (Soto Ballestero, 1992). Desse modo, a diferenciação floral deve ter ocorrido em condição desfavorável, refletindo posteriormente sobre o desenvolvimento do cacho. Os dados meteorológicos da região dão suporte a esta explicação (Figura 1). As maiores produções obtidas na terceira época (26/2/97) devem-se às melhores condições climáticas que ocorreram, desde a diferenciação floral nas bananeiras (novembro e dezembro/96) até o período de frutificação.

Não foram verificadas diferenças em relação à massa dos cachos ensacados e não ensacados. Resultados semelhantes foram obtidos por Sampaio \& Simão (1970), Daniells et al. (1992) e Salomão (1995) com as cultivares Nanica, Williams e Mysore, respectivamente. No entanto, Cann (1965), Robinson \& Nel (1984) e Galán Saúco et al. (1996) registraram aumentos significativos de massa em relação a cachos ensacados da cultivar Dwarf Cavendish.

Quanto às variáveis comprimento e diâmetro, são apresentados apenas os dados relativos à segunda penca superior, considerada como representativa do cacho, para efeito de uniformização de amostras (Carvalho et al., 1989; Galán Saúco et al., 1996), e indicada para determinação do ponto de colheita com base no diâmetro do fruto (Soto Balestero et al., 1992).

O comprimento do fruto foi maior na segunda (17/12/96) e terceira época (26/2/97) em relação ao verificado na primeira época de ensacamento (21/5/96). Não houve diferença significativa entre o comprimento dos frutos de cachos ensacados e não ensacados (Tabela 1).

Quanto ao diâmetro dos frutos da segunda penca superior, não foram observadas diferenças entre as médias de ensacamento, de épocas e da interação ensacamento x época. Em relação à densidade dos frutos, não foram detectadas diferenças significativas em relação aos fatores época e ensacamento (Tabela 1).

Singh et al. (1987), em estudo sobre crescimento e maturidade, encontraram valores de densidades de frutos variando de 0,970 a $0,993 \mathrm{~g} / \mathrm{cm}^{3} \mathrm{em}$ bananas
'Basrai', não ensacadas. Salomão (1995) constatou, densidades superiores a $1,00 \mathrm{~g} / \mathrm{cm}^{3}$ para 'Mysore', cujos cachos foram cobertos com envoltório plástico, e valores inferiores para frutos de cachos não ensacados. Conforme Lassoudière (1978), as variações de densidade estão relacionadas à cultivares, nível de nutrição potássica e ponto de colheita. Provavelmente, tais variações estejam relacionadas também às condições climáticas. Conforme Salomão (1995), deve existir um mecanismo fisiológico que atua sobre a expansão celular e o acúmulo de amido, o que faz preencher os espaços vazios das cavidades loculares.

\section{Conclusões}

1. O ensacamento realizado no início do verão propicia um menor período de dias entre a emergência da inflorescência e a colheita.

2. O ensacamento não aumenta a massa do cacho nem o comprimento e diâmetro dos frutos de banana.

\section{Referências}

ALVES, E. J.; OLIVEIRA, M. A.; DANTAS, J. L. L.; OLIVEIRA, S. L. Exigências climáticas. In: ALVES, E. J. (Org.). A cultura da banana: aspectos técnicos, socioeconômicos e agroindustriais. Brasília: Embrapa-SPI/ Embrapa-CNPMF, 1997. p. 335-351.

BERRIL, F. W. Bunch covers for bananas. Queensland Agricultural Journal, Brisbane, v. 82, n. 8, p. 53-62, 1956.

BLEINROTH, E. W. Matéria prima. In: INSTITUTO DE TECNOLOGIA DE ALIMENTOS (Campinas, São Paulo). Banana: cultura, matéria prima, processamento e aspectos econômicos. 2. ed. Campinas, 1985. p. 133-196.

CANN, H. J. Banana growing: plantation practices. Agricultural Gazette on New South Wales, Alstonville, v. 76, n. 11, p. 672-678, 1965.

CARVALHO, H. A. de; CHITARRA, M. I. F.; CARVALHO, H. S.; CHITARRA, A. B.; CARVALHO, V. D. Qualidade da banana 'Prata' previamente armazenada em filme de polietileno, amadurecida em ambiente com umidade relativa elevada. Pesquisa Agropecuária Brasileira, Brasília, v. 24, n. 5, p. 495-501, maio 1989.

DANIELLS, J. W.; LISLE, A. T.; O’FARRELL, P. J. Effect of bunch-covering methods on maturity bronzing, 
yield, and fruit quality of bananas in North Queensland. Australian Journal of Experimental Agriculture, Collingwood, v. 32, p. 122-125, 1992.

DANIELLS, J. W.; O'FARREL, P. J.; MULDER, J. C.; CAMPBELL, S. J. Effect of bunch covering and bunch trimming on bananas in North Queensland. Queensland Journal of Agricultural and Animal Sciences, Brisbane, v. 44, p. 101-105, 1987.

GALÁN SAÚCO, V. G.; CABRERA CABRERA, J. C.; GOMES LEAL, P. M. The evaluation of different bunch covers for bananas (Musa acuminata) in the Canary Islands. Fruits, Paris, v. 51, n. 1, p. 13-24, 1996.

GANRY, J. Influence du gainage des régimes de bananier avec une housse de polyéthylène, sur la température des fruits, dans les conditions de Neufchâteau (Guadeloupe). Fruits, Paris, v. 30, n. 12, p. 735-738, 1975.

HEENAN, D. P. Bunch covers for bananas in the Northern District. Papua New Guinea Agricultural Journal, Konedobu, v. 24, p. 156-161, 1973.

JOHNS, G. G.; SCOTT, K. J. Delayed harvesting of bananas with 'sealed' covers on bunches 2: effect on fruit yield and quality. Australian Journal of Experimental Agriculture, Collingwood, v. 29, p. 727-733, 1989.

KLUGE, R. A. Bananeira (Musa sp.). In: CASTRO, P. R. C.; KLUGE, R. A. (Ed.). Ecofisiologia de fruteiras tropicais: abacaxizeiro, maracujazeiro, mangueira, bananeira e cacaueiro. São Paulo: Nobel, 1998. p. 69-88.

LASSOUDIÈRE, A. Quelques aspects de la croissance et du développement du bananier 'Poyo' en Cote d'Ivoire. IV. L'inflorescence. Fruits, Paris, v. 33, p. 457-491, 1978.

LICHTEMBERG, L. A. Ensacamento do cacho de banana no campo. Informativo SBF, Brasília, n. 3, p. 8-11, set. 1996.

MEDINA, J. C. Cultura. In: INSTITUTO DE TECNOLOGIA DE ALIMENTOS (Campinas, São Paulo). Banana: Cultura, matéria-prima, processamento e aspectos econômicos. Campinas, 1985. p. 1-131.

MOREIRA, R. S. Banana: teoria e prática de cultivo. Campinas: Cargill, 1987. 335 p.

PERUMAL, A.; ADAM, A. V. Bagging of giant Cavendish banana stems in Honduras I: effect on number of days from flower emergence to fruit harvest. Tropical Agriculture, St. Augustine, v. 45, p. 109-112, 1968.

ROBINSON, J. C.; NEL, D. J. Influence of polyethylene bunch covers on yield and fruit quality of winter-developing banana bunches. Journal of Horticulture Science, The Hague, v. 1, p. 26-28, 1984.

SALOMÃO, L. C. C. Efeitos do envoltório plástico no desenvolvimento e na maturação pós-colheita de frutos de banana (Musa AAB) 'Mysore'. 1995. 104 f. Tese (Doutorado) - Universidade Federal de Viçosa, Viçosa, MG.

SAMPAIO, V. R.; SIMÃO, S. Banana: ensacamento de cachos logo após o florescimento. Revista de Agricultura, Piracicaba, v. 45, p. 75-77, 1970.

SCOTT, K. J.; WILLS, R. B. H.; RIPPON, L. E. The use of sealed polyethylene bunch covers during growth as a retardant to the ripening of bananas. Tropical Agriculture, St. Augustine, v. 48, p. 163-165, 1971.

SINGH, U. R.; GANGWUAR, B. M.; SINGH, G.; RAM, M. Growth and maturity studies on banana. Indian Journal of Horticulture, Bangalore, v. 33, p. 19-25, 1987.

SOTO BALLESTERO, M. Descripción botánica. In: SOTO BALLESTERO, M. (Ed.). Banano: cultivo y comercialización. San José: Litografía e Imprenta Lil, 1992. p. 21-104.

SOTO BALLESTERO, M.; SOTO, E.; SOLIS, P.; LÓPEZ, A. Siembra e operaciones de cultivo. In: SOTO BALLESTERO, M. (Ed.). Banano: cultivo y comercialización. San José: Litografía e Imprenta Lil, 1992. p. 211-365.

TURNER, D. W. Banana plant growth 1: gross morphology. Australian Journal of Experimental Agriculture and Animal Husbandry, Melbourne, v. 12, n. 55, p. 209-215, Apr. 1972a.

TURNER, D. W. Banana plant growth 2: dry matter production, leaf area and growth analysis. Australian Journal of Experimental Agriculture and Animal Husbandry, Melbourne, v. 12, n. 55, p. 216-224, Apr. 1972b.

TURNER, D. W.; RIPPON, L. E. Effect of bunch covers on fruit growth and maturity in bananas. Tropical Agriculture, St. Augustine, v. 50, p. 235-240, 1973. 